\title{
Representações Sociais da Qualidade de Vida na Velhice
}

Social Representations Of The

Quality Of Life In Old Age

Representaciones Sociales De La

Calidad De Vida En La Vejez

Kay Francis Leal Vieira, Isabella Dias dos Reis, João Bezerra de Morais

Segundo,

Magna Eugênia Fernandes

\& Talita Teilarda de

Vasconcelos Macdonald

Centro Universitário de João Pessoa

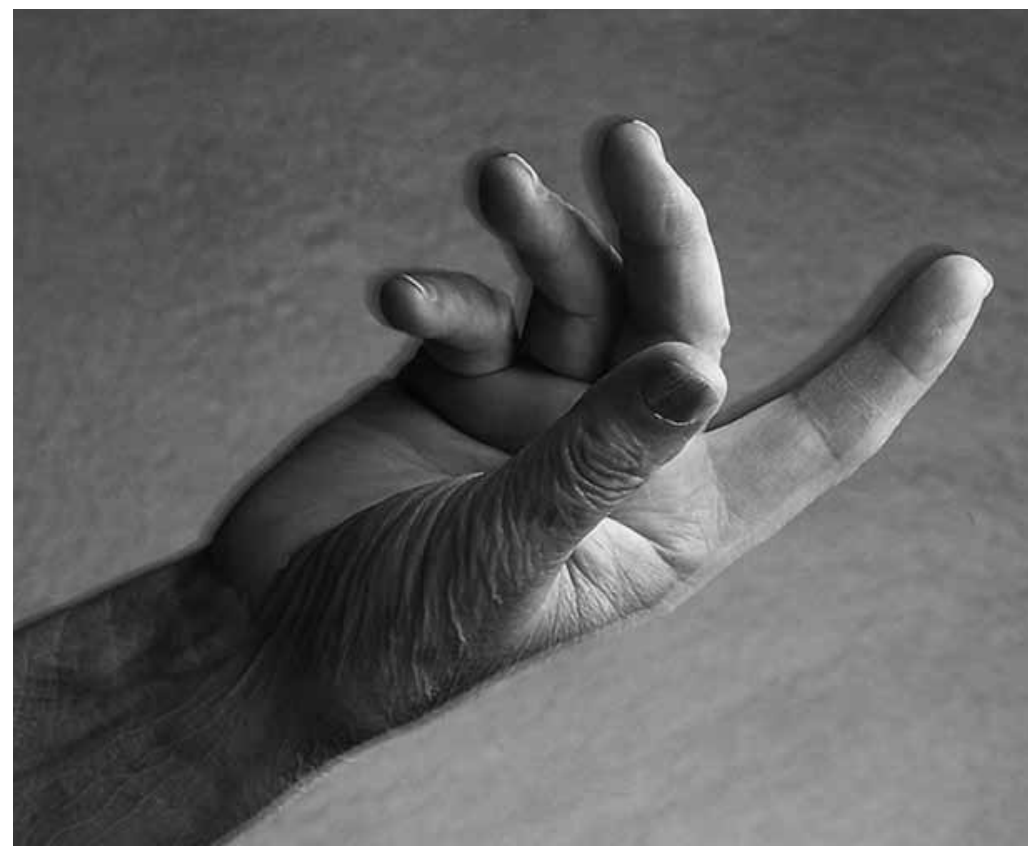


Resumo: É inegável o crescimento da população idosa nas últimas décadas, e surge a necessidade urgente de proporcionar a esse grupo condições biopsicossociais para uma qualidade de vida efetiva. Considerando esses aspectos, o presente estudo objetivou investigar a representação social da qualidade de vida elaborada pelos idosos. A amostra foi composta por 40 sujeitos, sendo 20 de instituição de longa permanência (ILP) e 20 frequentadores de um grupo de convivência localizado na cidade de João Pessoa - PB. Foi utilizada a entrevista em profundidade, sendo seu conteúdo submetido à análise de conteúdo temático. Os resultados apontaram semelhanças e divergências entre as suas representações, observando-se que os idosos de grupos de convivência representaram a qualidade de vida como algo que pertence a suas realidades, enquanto o segundo grupo a definiu em uma perspectiva do que lhes falta. Sendo assim, destaca-se a importância de proporcionar meios de incluir esse grupo no convívio social, ampliando as pesquisas e as ações que o beneficiem de forma integral.

Palavras-chave: Qualidade de vida. Representação social. Grupos etários. Instituição de longa permanência para idosos.

Abstract: There is no denying that the general population is getting increasingly older in recent decades and thus the urgent need to provide this group biopsychosocial conditions for an effective quality of life. Considering these aspects, this study focuses on the social representation of the quality of life as seen by the elderly. The sample consisted of 40 subjects, 20 who lived in a retirement home and 20 who attended support groups located in João Pessoa. The interview was carried out thoroughly, and its contents were subjected to thematic content analysis. The results showed similarities and differences between their representations, and that older people in social groups represented the quality of life as something that was part of their reality, while the second group defined it as something that lacks to them. Thus, we highlighted the importance of involving this group in social support groups, expanding the research and the importance of plans of actions that benefit them totally.

Keywords: Quality of life. Social representation. Developmental age groups. Homes for the aged.

Resumen: Es innegable el crecimiento de la población de edad avanzada en las últimas décadas, y surge la necesidad urgente de proporcionar a ese grupo condiciones bio-psico-sociales para una calidad de vida efectiva. Considerando esos aspectos, el presente estudio objetivó investigar la representación social de la calidad de vida elaborada por las personas de edad avanzada. La muestra fue compuesta por 40 sujetos, siendo 20 de institución de larga permanencia (ILP) y 20 frecuentadores de un grupo de convivencia localizado en la ciudad de João Pessoa - PB. Fue utilizada la entrevista en profundidad, siendo su contenido sometido al análisis de contenido temático. Los resultados apuntaron semejanzas y divergencias entre sus representaciones, observándose que las personas de edad avanzada de grupos de convivencia representaron la calidad de vida como algo que pertenece a sus realidades, mientras que el segundo grupo la definió en una perspectiva de lo que les falta. Siendo así, se destaca la importancia de proporcionar medios de incluir ese grupo en la convivencia social, ampliando las pesquisas y las acciones que lo beneficien de forma íntegra.

Palabras clave: Calidad de vida. Representación social. Grupos por edad. Hogares para ancianos.

Diante do processo inegável de envelhecimento populacional, surge a necessidade de proporcionar aos idosos não só uma sobrevida maior, mas, sobretudo, de melhor qualidade. O tema qualidade de vida na velhice, por sua abrangência e importância no mundo atual, tem sido objeto de muitos estudos e pesquisas, embora, como ressaltam Dalla Vecchia, Ruiz, Bocchi e Corrente (2005), esse conceito varie de autor para autor, representando algo subjetivo que depende do nível sociocultural, da faixa etária e das aspirações pessoais do indivíduo.

A temática da qualidade de vida tem sido abordada sob os mais diferentes olhares, tanto da ciência quanto do senso comum, e investigada do ponto de vista objetivo e do subjetivo. Apesar da existência de um consenso acerca da importância da avaliação da qualidade de vida, o mesmo não ocorre com o seu conceito, campo esse repleto de frequentes e significativos debates.

\section{O conceito qualidade de vida}

Segundo Fleck (2008), alguns autores reconhecem a complexidade e a impossibilidade de conceituar de forma adequada a qualidade de vida, tratando-a como uma variável emergente, da mesma forma que construtos como traços de 


\section{Barreto, Coutinho \\ e Ribeiro (2009) \\ ressaltam que \\ o conceito \\ qualidade \\ de vida é \\ polissêmico, \\ isto é, abrange \\ muitos \\ significados, \\ e reflete \\ conhecimentos, \\ experiências e \\ valores individuais \\ e coletivos, que \\ a ele se referem \\ em variadas \\ épocas, espaços \\ e histórias; \\ é, portanto, \\ um construto \\ social com \\ características \\ de relatividade \\ social.}

personalidade ou emoções, para os quais são atribuídos, preferencialmente, características ou indicadores que não merecem o status de definição. Dessa forma, a existência de diferentes definições decorreria, na verdade, do peso dado a cada uma dessas características ou indicadores.

Barreto, Coutinho e Ribeiro (2009) ressaltam que o conceito qualidade de vida é polissêmico, isto é, abrange muitos significados, e reflete conhecimentos, experiências e valores individuais e coletivos, que a ele se referem em variadas épocas, espaços e histórias; é, portanto, um construto social com características de relatividade social. Dessa forma, qualquer tentativa de forçar o enquadramento de tal conceito multidimensional em um único valor tende a falhar tanto por problemas conceituais quanto psicométricos de confiabilidade e validade (Power, 2008).

Neste estudo, considera-se que a definição proposta pela Organização Mundial de Saúde (OMS) é a que melhor traduz a abrangência desse construto, definindo-o como "a percepção do indivíduo de sua posição na vida, no contexto da cultura e do sistema de valores em que vive, e em relação a suas metas, expectativas, padrões e conceitos" (Fleck, 2008, p. 25). Tal percepção é afetada de forma complexa pela saúde física da pessoa, estado psicológico, crenças pessoais, relações sociais e por suas relações com características importantes de seu ambiente.

De acordo com Neri (2008), o termo qualidade de vida surgiu no final dos anos 50, sendo usado por sociólogos no contexto da discussão sobre poluição ambiental, deterioração da vida urbana e assuntos similares, veiculados em publicações não acadêmicas. No final da década de 70 , o tema começou a ser utilizado em eventos científicos internacionais e publicações em Sociologia e Medicina. Ainda segundo a autora supracitada, em 1985, esse termo apareceu pela primeira vez no Index Medicus e no Psychological Abstract.

Atualmente, a qualidade de vida é uma noção eminentemente humana avaliada mediante o grau de satisfação encontrado nos mais diversos campos da vida humana: familiar, amoroso, social e ambiental; segundo Cachioni e Falcão (2009), pressupõe a capacidade de efetuar uma síntese cultural de todos os elementos que determinada sociedade considera seu padrão de conforto e de bem-estar.

Segundo Neri (2008), na Medicina, o termo qualidade de vida é associado à relação custo - benefício inerente à manutenção da vida de enfermos crônicos e terminais, tanto do ponto de vista do bem-estar e dos direitos individuais quanto dos interesses e valores da sociedade. Em Economia, esse termo é associado à renda per capita, que funciona como um indicador do grau de acesso das populações aos benefícios da educação, da Medicina e dos serviços sociais. Já para a Sociologia, o conceito de qualidade de vida inclui um conjunto de indicadores econômicos e de desenvolvimento sociocultural identificados como nível ou padrão de vida de uma população. A referida autora destaca ainda que, em Psicologia social, a referência mais forte é a experiência subjetiva de qualidade de vida, representada pelo conceito de satisfação.

Nessa ótica da subjetividade, Coutinho, Franken e Ramos (2007) afirmam que qualidade de vida não é o reflexo direto das condições reais e objetivas da vida das pessoas, mas da avaliação que cada um faz a respeito dessas condições. Dessa forma, tem-se os elementos subjetivos, entendidos como não materiais (amor, liberdade, inserção e realização social), e os objetivos representativos dos elementos materiais (alimentação, habitação, educação, trabalho, 
saúde e lazer), que têm como referência noções relativas ao conforto, ao bem-estar e à realização individual e coletiva.

Descobrir virtudes na velhice, prolongar a juventude e envelhecer com qualidade de vida individual e social têm sido preocupações sistemáticas do ser humano, manifestadas em diversas áreas do conhecimento, e a preocupação com a qualidade de vida na velhice ganhou relevância nas últimas décadas, a partir do crescimento do número de idosos e da expansão da longevidade. Segundo Neri (2008), cada vez mais as questões que dizem respeito ao bemestar físico, psicológico e social dos idosos interessam aos planejadores de políticas de saúde, educação, trabalho e seguridade social, bem como aos cientistas.

A qualidade de vida na velhice parte de uma avaliação multidimensional em relação aos critérios socionormativos e intrapessoais que buscam referência tanto nas relações atuais quanto nas passadas e também nas prospectivas na relação entre o idoso e o ambiente que o cerca. Dessa forma, conforme ressaltam Trentini, Chachamovich e Fleck (2008), a qualidade de vida na velhice depende de muitos elementos em interação constante ao longo da vida do indivíduo.

Face a essas premissas, torna-se indispensável a investigação das representações sociais da qualidade de vida para a pessoa idosa, uma vez que elas se referem a "uma forma de conhecimento, socialmente elaborada e partilhada, com um objetivo prático, e que contribui para a construção de uma realidade comum a um conjunto social" (Jodelet, 2001, p. 22).

\section{Representações sociais}

A teoria das representações sociais é uma forma sociológica de Psicologia social, originada na Europa e desenvolvida por
Serge Moscovici através da publicação de seu estudo La Psychanalyse: son Image et son Public (1961). Segundo Farr (1994), ela difere, marcadamente, das formas psicológicas de Psicologia social que são atualmente predominantes nos Estados Unidos da América. Esse contraste ocorre entre uma tradição de pesquisa européia e uma americana de Psicologia social moderna.

De acordo com Moscovici (1978), a representação social, como modalidade de conhecimento particular, tem por função orientar comportamentos e facilitar a comunicação entre os indivíduos, considerando a indissociabilidade entre a experiência subjetiva e a inserção social dos sujeitos. Por conseguinte, as representações sociais dos idosos sobre a qualidade de vida podem ser compreendidas como uma interpretação da realidade vivida e falada por esse grupo social, que direciona comportamentos e comunicações.

A elaboração e o funcionamento de uma representação podem ser compreendidos por meio dos processos de objetivação e ancoragem. Jodelet (2001) afirma que a objetivação é o processo pelo qual o indivíduo reabsorve um excesso de significações, materializando-as, ou seja, é a construção formal de um conhecimento pelo indivíduo. Já a ancoragem, como instrumento do saber, é uma modalidade que permite compreender como os elementos de representação não só exprimem relações sociais mas também como contribuem para construí-las. Ela assegura, portanto, o elo entre a função cognitiva de base da representação e a social, bem como fornece à objetivação os elementos imaginativos para servir na elaboração de novas representações.

Segundo Vala e Monteiro (2004), as representações sociais apresentam uma dimensão funcional e prática que acaba por ser evidente na organização 
dos comportamentos, das atividades comunicativas, na argumentação e na explicação cotidianas e na diferenciação dos grupos sociais. Desse modo, identificar as representações sociais acerca da qualidade de vida é compreender as formas que as pessoas utilizam para criar, transformar e interpretar esse construto vinculado à sua realidade.

Nessa perspectiva, a presente pesquisa objetivou apreender as representações sociais da qualidade de vida elaboradas por idosos inseridos em instituições de longa permanência e idosos frequentadores de grupos de convivência localizados no Município de João Pessoa, PB. A comparação entre os grupos se faz relevante, uma vez que há diferentes vivências e rotinas que corroboram o diferente nivelamento e a representação da qualidade de vida. Considerando que a representação social é algo construído nas trocas de conhecimentos populares e científicos, através de experiências grupais e sociais adquiridas ao longo da vida, acredita-se que os idosos inseridos em contextos tão distintos possuam diferentes representações acerca da qualidade de vida.

\section{Método}

Esta é uma pesquisa descritiva com abordagem qualitativa, fundamentada nos aportes teóricos e metodológicos da teoria das representações sociais (Moscovici, 1978). A amostra foi do tipo não probabilístico, intencional e acidental, constituída por 40 idosos de ambos os sexos, estando 20 inseridos em uma ILP e 20 frequentadores de grupo de convivência, ambos localizados no Município de João Pessoa, PB. Devido ao alto índice de comprometimento cognitivo, contou-se com o auxílio de funcionários da ILP, no sentido de fornecer informações acerca dos idosos que teriam condições neuropsicológicas de responder aos instrumentos.
A maioria dos participantes foi do sexo feminino $(72,5 \%)$, e suas idades variaram de 60 a 85 anos $(M=70,3$ e $D P=5,29)$. No que concerne ao estado civil, verificou-se que $32,5 \%$ eram casados; $27,5 \%$, solteiros; $32,5 \%$, viúvos, e 7,5\%, divorciados. Em relação à religião, 47,5\% dos idosos afirmaram ser católicos; $37,5 \%$, evangélicos; 7,5\%, espíritas, e $7,5 \%$ afirmaram não possuir nenhuma religião.

Foram utilizados como instrumentos de coleta dos dados um questionário biossociodemográfico e a entrevista em profundidade, entendida como aquela em que o entrevistado tem a possibilidade de discorrer sobre o tema proposto, sem respostas ou condições prefixadas pelo pesquisador (Minayo, 1998). Durante a entrevista, os idosos foram convidados a falar de forma livre e aberta sobre sua compreensão acerca da qualidade de vida. O questionário biossociodemográfico foi elaborado pelos pesquisadores com a finalidade de se obter informações relevantes acerca dos participantes da pesquisa, tais como o tipo de instituição, sexo, idade, estado civil e religião.

Este estudo foi aprovado pelo Comitê de Ética em Pesquisa do Centro Universitário de João Pessoa - UNIPE. Os participantes foram previamente informados a respeito dos objetivos e dos procedimentos da pesquisa, bem como da confiabilidade dos dados e do anonimato da sua colaboração. Em seguida, foi solicitado que assinassem o Termo de Consentimento Livre e Esclarecido, de acordo com as normas da Resolução no 196/96 sobre Pesquisa Envolvendo Seres Humanos.

A coleta de dados foi realizada de forma individual nas instituições às quais os idosos estavam vinculados, ou seja, ILP e grupo de convivência. As entrevistas foram gravadas com o consentimento dos participantes e seu conteúdo submetido à análise de conteúdo temático, entendida como um conjunto de técnicas de análise das comunicações que 
visa a obter indicadores que permitam a inferência de conhecimentos relativos às condições de produção/recepção das mensagens (Bardin, 2002).

\section{Resultados e discussão}

Com base no discurso dos idosos, emergiram quatro categorias empíricas com variações apenas no que concerne às subcategorias, sendo 16 para os idosos em contexto asilar e 12 para os frequentadores do grupo de convivência, conforme descrição a seguir.

Tabela 1. Representações sociais da qualidade de vida elaboradas por idosos

\begin{tabular}{|c|c|c|c|}
\hline & & $\begin{array}{l}\text { INSTITUIÇÃO DE LONGA } \\
\text { PERMANÊNCIA }\end{array}$ & GRUPO DE CONVIVÊNCIA \\
\hline \multirow{8}{*}{$\begin{array}{l}\text { CLASSE } \\
\text { TEMÁTICA }\end{array}$} & CATEGORIAS & SUBCATEGORIAS & SUBCATEGORIAS \\
\hline & 1. ASPECTOS & 1. Saúde & 1. Saúde \\
\hline & FísıCOS & 2. Alimentação & 2. Alimentação \\
\hline & & 3. Vestuário & \\
\hline & & 4. Descanso/ & \\
\hline & & repouso & \\
\hline & 2. ASPECTOS & 1. Felicidade & 1. Felicidade \\
\hline & PSICOLÓGICOS & 2.Autoestima & 2. Independência \\
\hline \multirow{9}{*}{$\begin{array}{l}\text { QUALIDADE } \\
\text { DE VIDA NA } \\
\text { VELHICE }\end{array}$} & & 1. Lar & 1. Lar \\
\hline & & 2. Família & 2. Família \\
\hline & 3. ASPECTOS & 3. Lazer & 3. Lazer \\
\hline & SOCIAIS & 4. Amizade & 4. Esporte \\
\hline & & 5. Trabalho & 5. Amizade \\
\hline & & 6. Dinheiro & 6. Trabalho \\
\hline & & 7. Cuidador & 7. Solidariedade \\
\hline & 4. ASPECTOS & 1. Paz & 1. Paz \\
\hline & ESPIRITUAIS & 2. Crença & 2. Crença \\
\hline
\end{tabular}

Com relação aos aspectos físicos, constatou-se que, para ambos os grupos, a saúde representa um fator necessário e foi apontada pelos idosos do grupo de convivência como algo a ser prevenido (...é ter saúde, ...se manter saudável, ...se cuidar, ...ir regularmente ao médico...), enquanto, para os idosos da instituição asilar, corresponde a algo que lhes falta (...é não ter doença, ...era se a gente não tiver essas doenças, ...dói aqui, dói acolá...). Nesse sentido, a representação social da qualidade de vida assume um contorno diferenciado entre os grupos, e, para as pessoas desse último grupo, é algo que julgam não possuir.

É inegável o papel da saúde física na promoção da qualidade de vida do indivíduo, existindo, atualmente, algumas evidências científicas que demonstram a contribuição da saúde para a qualidade de vida do idoso. Vilar (2000) ressalta que o envelhecimento populacional transformou o perfil do adoecimento dessa população, que está cada vez mais acometida por doenças crônicas de alta morbimortalidade e incapacitação. As doenças crônicas são atreladas geralmente a sentimentos de vergonha e culpa, que trazem consequências negativas sobre a percepção da qualidade de vida dos indivíduos acometidos e seus familiares. Para Pereira, Cotta, Franceschini, Ribeiro, 
Patrick (2008)

afirma que há

considerável

concordância de

que o construto

qualidade de

vida é mais

abrangente do

que o estado de

saúde, sendo

este apenas um

de seus domínios.
Sampaio e Priore (2006), quando se investiga a qualidade de vida relacionada à saúde em sua multidimensionalidade, identificam-se os principais aspectos a serem considerados em relação às potencialidades e às peculiaridades de saúde e vida do idoso que interferem no seu processo saúde-doença.

Patrick (2008) afirma que há considerável concordância de que o construto qualidade de vida é mais abrangente do que o estado de saúde, sendo este apenas um de seus domínios. Nesse sentido, afirma que a qualidade de vida inclui aspectos do ambiente que podem ou não ser afetados pela saúde do indivíduo ou pela saúde por ele percebida.

A alimentação foi fator comum também na representação da qualidade de vida do idoso, embora, para os frequentadores do grupo de convivência, esteja mais relacionada à qualidade da refeição (...é comer bem, ... é ter uma dieta balanceada, ...é ter boa alimentação), não se limitando ao simples fato de comer, como ocorreu com os idosos institucionalizados. (...é ter o que comer, ...é ter comida...).

A qualidade da alimentação dos idosos é de suma importância, uma vez que é alto o risco de desnutrição nessa fase da vida, principalmente quando se trata de idosos institucionalizados. Segundo Santelle, Lefevre e Cervato (2007), as refeições nas instituições de longa permanência para idosos poderiam ser melhoradas através do planejamento de cardápios que contemplem as preferências alimentares dos idosos e que atendam às suas necessidades nutricionais. As autoras ressaltam, ainda, a necessidade de atenção especial para os indivíduos que necessitam de alimentação assistida, que possibilitaria um estado nutricional equilibrado e melhor qualidade de vida para esses sujeitos.
Complementando os aspectos físicos da qualidade de vida na velhice, emergiram ainda, por parte dos idosos institucionalizados, duas outras categorias, a primeira, relacionada ao vestuário (...é ter o que vestir, ...é ter suas roupinhas tudo organizadinhas...), e a segunda ressalta o descanso e o repouso como indicativos de qualidade de vida (...é ter repouso, ...é poder ficar no seu cantinho...).

Observou-se que a representação da qualidade de vida elaborada pelos idosos institucionalizados relacionada ao vestir não está limitada a uma simples referência ao objeto vestuário, mas apresenta-se com um significado subjetivo de autoestima, de autopercepção, de autonomia, de identidade. Muitas vezes, o idoso institucionalizado tem sua autonomia de escolha do vestuário reduzida, tendo em vista que, em algumas ILPIs, como é o caso desta onde foi realizado o estudo, há uma padronização do vestir por meio do fardamento, que, de certa forma, desfavorece o aspecto da identidade que a roupa exerce. Em outras instituições, onde não há padronização das roupas, ainda assim o idoso tem sua autonomia reduzida, pois não pode ausentar-se da ILP para adquirir seu vestuário a sua maneira e/ou porque esse vestuário é limitado ao conjunto de peças advindas de doações de terceiros e de aquisições feitas por familiares.

Em relação ao aspecto do descanso e do repouso, também evidenciado apenas pelos idosos institucionalizados, pode-se inferir que o processo de objetivação da representação da qualidade de vida, transformada na imagem do descanso, do repouso, remonta à necessidade, para esses idosos, de um envelhecimento tranquilo, sem perturbações.

No que concerne à categoria aspectos psicológicos, observou-se que as representações sociais foram ancoradas em conceitos abstratos e subjetivos como 
a felicidade (...é ser feliz, ...ter alegria de viver, ...ser alegre sem aquela coisa no coração, ...ter sempre um sorriso no rosto...), representada por ambos os grupos de idosos, a independência (...é você poder ir aonde quer, ...fazer suas coisas, ...não depender dos outros...), para os idosos de grupos de convivência, e a autoestima (...é você se arrumar, ...se sentir bonita, ...gostar da gente mesmo...), para os institucionalizados.

A promoção da felicidade, relatada como importante para os idosos, é algo proposto como objetivo básico dos grupos de convivência que, de acordo com Ferrigno, Leite e Abigail (2006), propicia a busca da realização pessoal e de sonhos comuns. Outra proposição básica é a promoção do exercício da cidadania, que capacita o idoso a sentir-se integrado e ativo, o que colabora para o seu senso de independência.

Avaliando os aspectos sociais, foi possível identificar algumas categorias comuns, tais como o lar (...é ter sua casa, é ter onde morar...), a família (...é ter família, ...é você viver bem com sua família...), amizade (...é a gente ter amigo, ... fazer amizade, ... precisa cultivar seus amigos) e lazer (...ter lazer, ...participar de festas, ... passear). Percebese, em ambos os grupos, uma valorização do outro, seja os familiares, seja os amigos, como meio de socialização e de interação social.

De acordo com Prado e Perracini (2007), envelhecer no próprio lar e na própria comunidade é um desejo tanto das pessoas idosas quanto de seus familiares, e é apontada pela literatura gerontológica dos últimos 30 anos como uma das metas a serem alcançadas para a promoção de um envelhecimento ativo. Entende-se que o lar não é caracterizado apenas por um ambiente físico, mas, especialmente, pelas pessoas que ali residem, pelos sentimentos que existem entre os moradores e pelas lembranças dos momentos vividos naquele lugar.
Algumas subcategorias apresentaram significados divergentes para os dois grupos. No que diz respeito aos aspectos trabalho e dinheiro, observou-se que o grupo da instituição de longa permanência os representou como algo ligado a independência e autonomia, relacionandoos com a qualidade de vida no sentido de ter domínio (...é você ter dinheiro, ...ter o seu sustento, ...trabalhar e ganhar seu dinheiro...). Já nos grupos de convivência, a preocupação com a situação financeira não é evidenciada, mas apenas a questão do trabalho em si, como medida ocupacional (...é você poder trabalhar, ...ter seu emprego, ... uma ocupação para se sentir útil...).

Segundo Wajnman, Oliveira e Oliveira (2004), atualmente, há uma tendência do crescimento da participação dos idosos no mercado de trabalho brasileiro e, em consequência, surge a necessidade da criação de políticas de emprego focadas nessa população. Compreende-se aqui que o trabalho não é meramente uma forma de se obter dinheiro, mas, principalmente, um instrumento de promoção de autoestima, independência e autonomia, extremamente importantes para a qualidade de vida da pessoa idosa.

A subcategoria lazer também apresentou divergências entre os grupos pesquisados, sendo que os idosos não institucionalizados a trazem mais como uma medida para conhecer pessoas, como diversão, convivência (...passear, ...conhecer pessoas novas, ...conversar com as amigas...), enquanto os institucionalizados a retratam mais como um passeio (...poder passear, ...poder sair e ver gente...).

A formação de grupos de convivência constitui uma das formas de agregar e socializar a pessoa idosa, prática essa que tem se difundido em todas as localidades do País. A frequência dos idosos nos grupos 
é de extrema importância, haja vista que o convívio social promove a manutenção e a ampliação do seu grupo de amizades, o que poderá transmitir maior segurança e suporte social. Moura, Leite e Hildebrandt (2008) ressaltam ainda que, nesse cenário, há maior aproximação entre os idosos e a formação de novos vínculos afetivos.

A subcategoria esporte emergiu das representações sociais elaboradas pelos idosos frequentadores de grupos de convivência, em que os mesmos ressaltaram a prática de exercícios físicos como um quesito importante para a promoção da qualidade de vida (... praticar esportes, ... fazer atividade física com regularidade, ...fazer uma caminhada...).

Nesse sentido, os grupos de convivência são de extrema importância, pois, na maioria dos casos, possibilitam a seus membros a prática de atividades físicas regulares. Em estudo sobre o assunto, Mota, Ribeiro, Carvalho e Matos (2006) encontraram associação com alta qualidade de vida no grupo de idosos praticantes de atividades em comparação aos sedentários. Em contrapartida, os estudos de Vieira (1996) mostraram que as instituições de longa permanência são ambientes que não favorecem as relações interpessoais e a participação dos idosos nos seus contextos comunitários, havendo nesses locais uma propensão para os sentimentos de isolamento e para a inatividade física e mental.

A subcategoria solidariedade emergiu apenas no discurso dos idosos frequentadores do grupo de convivência, em que foi destacada a importância de ajudar o outro como meio de qualidade de vida (...é fazer bem ao próximo, ....judar a quem você nem conhece, ...poder ajudar a quem precisa...). Já os idosos de instituição de longa permanência destacaram a importância de um cuidador, de serem ajudados (...é ter quem cuide da gente, ...quando tem alguém que se importa e possa cuidar...).
Observou-se, no discurso dos idosos, uma oposição significativa no que diz respeito à representação da qualidade de vida, que, para os idosos não institucionalizados, significa o auxílio que os mesmos possam prestar aos necessitados. Por outro lado, os idosos da ILP se auto-representaram como esses necessitados, e ressaltaram a importância do trabalho de um cuidador.

No que concerne os aspectos espirituais, podemos perceber que a paz é um item comum (...é ter paz com a família, ... é viver em paz, ...ter paz na sua vida...), assim como a crença em Deus (....acreditar em Deus, ...ter fé em Deus, ...viver com Deus...), embora essa crença tenha sido mais enfatizada no discurso do idosos dos grupos de convivência.

De fato, os aspectos espirituais são considerados uma importante dimensão da qualidade de vida do idoso. Conforme ressaltam Panzini, Rocha, Bandeira e Fleck (2007), apesar da falta de um consenso geral sobre os conceitos, a literatura científica tem demonstrado a existência de relação entre espiritualidade e qualidade de vida, chegando a desenvolver instrumentos válidos e confiáveis para acessá-la e para produzir inúmeros estudos com rigor metodológico.

\section{Considerações finais}

As representações sociais da qualidade de vida na velhice elaboradas pelos idosos de ambos os grupos apresentaram-se de forma semelhante à descrição científica, demonstrando similaridades entre o senso comum e o conhecimento erudito. Foram identificados os aspectos físicos, psicológicos, sociais e espirituais, sendo possível perceber algumas semelhanças e divergências nas representações dos grupos investigados.

Através da análise dos dados, foi possível obter uma análise científica do que se chama senso comum. Essa análise se baseou no pressuposto de que o conhecimento do 
senso comum permite-nos compreender o construto qualidade de vida a partir de um conhecimento socialmente elaborado e partilhado. Por meio do discurso dos idosos, foi possível refletir sobre a dimensão do fenômeno pesquisado bem como sobre a percepção subjetiva deste em suas vidas.

O discurso dos idosos acerca da qualidade de vida na velhice foi permeado de conhecimentos oriundos do senso comum, interligados a experiências de vida dos atores sociais, e assumiu um contorno diferenciado em relação aos grupos de pertença: para os idosos institucionalizados, é algo que Ihes falta, que perderam ou que buscam, e, para os idosos frequentadores do grupo de convivência, as representações foram baseadas em seu cotidiano, sendo enfatizadas as atividades e os acontecimentos diários.

Acredita-se que este estudo possa promover reflexões e mudanças na assistência à população idosa, em especial a inserida em instituições de longa permanência, que necessita de maior atenção no que se refere à sua saúde mental e ao bem-estar biopsicossocial. Entretanto, são reconhecidas as limitações da pesquisa, bem como a complexidade do fenômeno investigado, salientando-se ainda a necessidade de novas pesquisas nas áreas da Psicologia e gerontologia objetivando-se a mensuração e a posterior comparação da qualidade de vida dos idosos em instituições de longa permanência e em grupos de convivência. 


\section{Kay Francis Leal Vieira}

Doutora em Psicologia Social pela Universidade Federal da Paraíba, docente do Centro Universitário de João

Pessoa - UNIPE, João Pessoa - PB - Brasil.

E-mail: kayvieira@yahoo.com.br

Isabella Dias dos Reis

Acadêmica do curso de Psicologia no Centro Universitário de João Pessoa, João Pessoa - PB - Brasil.

E-mail: isabelladiasdosreis@gmail.com

\section{João Bezerra de Morais Segundo}

Acadêmico de Psicologia do Centro Universitário de João Pessoa, João Pessoa - PB - Brasil.

E-mail: jbms.psi@gmail.com

\section{Magna Eugênia Fernandes}

Acadêmica de Psicologia do Centro Universitário de João Pessoa - PB - Brasil.

E-mail: magna.psi@gmail.com

Talita Teilarda de Vasconcelos Macdonald

Acadêmica de Psicologia do Centro Universitário de João Pessoa - PB - Brasil.

E-mail: talita_mac@hotmail.com

Endereco para envio de correspondência:

Rua Golfo de Biscaia, 36, ap. 401 Intermares Cabedelo, Paraiba - PB. CEP: 58310-000

Recebido 21/7/2011, 1a Reformulação 15/10/2011, Aprovado 10/1/2012. 
Barreto, L. M. S., Coutinho, M. P. L., \& Ribeiro, C. G. (2009). Qualidade de vida no contexto migratório: um estudo com imigrantes africanos residentes em João Pessoa, PB. Mudanças - Psicologia da Saúde, 17(2), 116-122.

Cachioni, M., \& Falcão, D. V. S. (2009). Velhice e educação: possibilidades e benefícios para a qualidade de vida. In D. V. S. Falcão \& L. F.Araújo (Orgs.), Psicologia do envelhecimento: relações sociais, bem-estar subjetivo e atuação profissional em contextos diferenciados (pp. 175-194). Campinas, SP: Alínea.

Coutinho, M. P. L., Franken, I., \& Ramos, N. (2007). Migração e qualidade de vida: o pensamento de brasileiros imigrantes. In E. C. Krutzen \& S. B. Vieira (Orgs.), Psicologia social, clínica e saúde mental (pp. 160-179). João Pessoa, PB: Ed. Universitária - UFPB.

Dalla Vecchia, R., Ruiz, T., Bocchi, S. C. M., \& Corrente, J. E. (2005). Qualidade de vida na terceira idade: um conceito subjetivo. Revista Brasileira de Epidemiologia, 8(3), 246-252.

Farr, R. M. (1994). Representações Sociais: A teoria e sua história. In P. Guareschi \& S. Jovchelovtih (Orgs), Textos em Representações Sociais (pp.31-55). Petrópolis, RJ: Vozes.

Ferrigno, J. C., Leite, M. L. C. B., \& Abigail, A. (2006). Centros e grupos de convivência de idosos: da conquista ao lazer ao direito ao exercício da cidadania. In E. L. Freitas, L. Py, F. A X. Cançado, J. Doll, M. L. Gorzoni \& S. M. Rocha (Orgs.) Tratado de geriatria e gerontologia (pp. 1436-1443). Rio de Janeiro: Guanabara Koogan.

Fleck, M. P. A. (2008). Problemas conceituais em qualidade de vida. In M. P. A. Fleck (Org.), A avaliação da qualidade de vida: guia para profissionais da saúde. Porto Alegre: Artmed.

Jodelet, D. (2001). Representações sociais: um domínio em expansão. In D. Jodelet (Org.), As representações sociais (pp.17-41). Rio de Janeiro: Ed. UERJ.

Minayo. M. C. S. (1998). O desafio do conhecimento: pesquisa qualitativa em saúde (5a. ed.). São Paulo: Hucitec/Abrasco.

Moscovici, S. (1961). Psychanalyse, son image et son public: étude sur la representation sociale de la psychanalyse. Paris: Press Universitaires de France.

Moscovici, S. (1978). A representação social da psicanálise. Rio de Janeiro: Zahar.

Mota, J., Ribeiro, J. L., Carvalho, J., \& Matos, M. G. (2006). Atividade física e qualidade de vida associada à saúde em idosos participantes e não participantes em programas regulares de atividade física. Revista Brasileira de Educação Física e Esporte, 20(3), 219-225.
Moura, I, Leite, M. T, \& Hildebrandt, L. M. (2008). Idosos e sua percepção acerca da sexualidade na velhice. $R B C E H$, 5(2), 132-140.

Neri, A. L. (2008). Palavras-chave em gerontologia. Campinas, SP: Alínea.

Panzini, R. G., Rocha, N. S., Bandeira, D. R., \& Fleck, M. P. A. (2007). Qualidade de vida e espiritualidade. Revista de Psiquiatria Clínica, 34, 105-115.

Patrick, D. L. (2008). A qualidade de vida pode ser medida? Como? In M. P. A. Fleck et al. A avaliação de qualidade de vida: guia para profissionais da saúde. Porto Alegre: Artes Médicas.

Pereira, R. J., Cotta, R. M. M., Franceschini, S. C. C., Ribeiro, R. C. L, Sampaio, R. F., \& Priore, S. E. (2006). Contribuição dos domínios físico, social, psicológico e ambiental para a qualidade de vida global de idosos. Revista de Psiquiatria do Rio Grande do Sul, 28(1), 27-38.

Power, M. (2008). Qualidade de vida: visão geral do projeto WHOQOL. In M. P. A. Fleck et al. (2008). A avaliação de qualidade de vida: guia para profissionais da saúde. Porto Alegre: Artmed.

Prado, A. R.de A., \& Perracini, M. R. (2007). A construção de ambientes favoráveis ao idoso. In A. L. Néri (Org.), Qualidade de vida na velhice: enfoque multidisciplinar. Campinas, SP: Alínea.

Santelle, O., Lefevre, A. M. C., \& Cervato, A. M. (2007). Alimentação institucionalizada e suas representações sociais entre moradores de instituições de longa permanência para idosos em São Paulo, Brasil. Cadernos de Saúde Pública, 23(12), 3061-3065.

Trentini, C. M., Chachamovich, E., \& Fleck, M. P. A. (2008). Qualidade de vida em idosos. In M. P. A. Fleck (Org.), A avaliação da qualidade de vida: guia para profissionais da saúde. Porto Alegre: Artmed.

Vala, J., \& Monteiro, M. B. (2004). Psicologia social. Lisboa: F.C. Gulbenkian.

Vieira, E. B. (1996). Manual de gerontologia: um guia teóricoprático para profissionais cuidadores e familiares. Rio de Janeiro: Revinter.

Vilar, J. M. (2000). A crise no setor saúde e o ranking da OMS Revista Brasileira de Home Care, 6(22).

Wajnman, S., Oliveira, E., \& Oliveira, A. M. (2004). Os idosos no mercado de trabalho: tendências e conseqüências. In A. A. Camarano (Org.), Os novos idosos brasileiros: muito além dos 60? (pp. 453-480). Rio de Janeiro: Ipea. 